\title{
Transatlantica
}

Revue d'études américaines. American Studies Journal

\section{Débat étatsunien sur les statistiques ethno- raciales : l'exemple de la Proposition 54 en Californie}

\section{Olivier Richomme}

\section{(2) OpenEdition}

1 Journals

\section{Édition électronique}

URL : http://journals.openedition.org/transatlantica/5276

DOI : $10.4000 /$ transatlantica. 5276

ISSN : 1765-2766

Éditeur

AFEA

\section{Référence électronique}

Olivier Richomme, « Débat étatsunien sur les statistiques ethno-raciales : l'exemple de la Proposition 54 en Californie », Transatlantica [En ligne], 1 | 2011, mis en ligne le 03 décembre 2011, consulté le 29 avril 2021. URL : http://journals.openedition.org/transatlantica/5276 ; DOI : https://doi.org/10.4000/ transatlantica.5276

\section{Ce document a été généré automatiquement le 29 avril 2021.}

\section{c) $(1)$}

Transatlantica - Revue d'études américaines est mis à disposition selon les termes de la licence Creative Commons Attribution - Pas d'Utilisation Commerciale - Pas de Modification 4.0 International. 


\title{
Débat étatsunien sur les statistiques ethno-raciales : l'exemple de la Proposition 54 en Californie
}

\author{
Olivier Richomme
}

\section{Les paradoxes de la démocratie directe : l'occasion manquée de la Proposition 54}

Alors que la polémique continue en France concernant l'éventualité de créer des statistiques ethno-raciales, il parait opportun de s'intéresser aux problématiques développées dans les pays qui possèdent déjà un tel système. Aux États-Unis, ce qui caractérise le débat concernant ces statistiques est le très faible niveau de contestation de leur omniprésence. Alors que les politiques antidiscriminatoires sont loin de faire l'unanimité, il semble exister un consensus quand à la nécessité de classer les Américains suivant des catégories ethniques et/ou raciales ${ }^{1}$. Les occasions de voir émerger un débat public sur la question sont extrêmement rares et ponctuelles. Toutefois, en 2003, lors d'un référendum d'initiative populaire, les électeurs californiens ont dû, pour la première fois, se prononcer sur la question épineuse de l'utilité de cette classification ethno-raciale. Cette campagne électorale nous éclaire sur les thèmes et enjeux du débat étatsunien concernant ces statistiques et les politiques antidiscriminatoires qui en dépendent. Mais surtout elle illustre les difficultés à ouvrir un débat de fond sur la question de la taxonomie ethno-raciale. Cette question légitime, d'un intérêt politique majeur, reste marquée par des clivages partisans et n'arrive pas à faire jaillir une réflexion d'ensemble sur la représentation identitaire et politique même lorsque l'occasion se présente. Loin de penser que cette forme de classification des individus au sein d'une société est a priori dangereuse, l'opinion américaine se distingue plutôt par un tabou inverse, à savoir l'impossibilité de penser l'absence de classification et l'impossibilité discursive de faire émerger un débat public sur la légitimité de cette classification ${ }^{2}$. En effet, les statistiques ethno-raciales existent 
depuis la fondation des États-Unis, notamment à cause du rôle centrale de l'esclavage dans le contrat social et économique de la jeune république, et font partie des institutions politiques et de la tradition administrative de gouvernance.

En Californie, ce sont les conservateurs, notamment l'American Institute for Civil Rights et son fondateur Ward Connerly, fort des succès de leurs référendums d'initiative populaire qui mirent fin à l'affirmative action dans le domaine public en Californie en 1996, avec la Proposition 209, et en 1998 dans l'État de Washington, qui innovèrent et tentèrent d'amender la Constitution de l'État afin de rendre illégales certaines formes de classification ethno-raciale émanant des pouvoirs publics. Pour ce faire l'institut concocta un amendement qui, après avoir rassemblé plus d'un million de signatures, fut soumis au Californiens sous la forme d'un référendum d'initiative populaire lors de l'élection anticipée de $2003^{3}$. Initialement appelé CRECNO, (acronyme pour «Classifying by Race, Ethnicity, Color and National Origin»), ce texte fut habilement rebaptisé «Racial Privacy Initiative ", soit, pour être clair, un référendum pour la protection de l'« intimité raciale » ou du droit de ne pas faire de son identité ethno-raciale une chose publique. Cette reformulation est intéressante car elle joue sur le thème de l'intime qui est très mobilisateur pour l'aile la plus conservatrice du parti républicain au sein de laquelle l'intervention des pouvoir publics et de l'État est perçue comme une invasion de la sphère privée ${ }^{4}$. Plusieurs mois avant l'élection, et surtout avant la campagne éclair et peu conventionnelle de l'élection qui allait par ailleurs destituer le gouverneur Gray Davis de son poste, la Proposition était donnée gagnante par certains instituts de sondage avec une avance confortable (48\% contre 33\%). Jusqu'en septembre 2003, la Proposition 54 était en tête dans le sondage multilingue des votants de Californie, même au sein de chaque groupe ethno-racial ${ }^{5}$. En effet, les «Latinos » étaient $46 \%$ à être en faveur du projet d'amendement de la constitution alors que $33 \%$ se déclaraient contre. Ces chiffres étaient de $41 \%$ favorables et $33 \%$ défavorables chez les «Africainsaméricains ", $31 \%$ pour et $25 \%$ contre chez les Blancs et $42 \%$ pour et $40 \%$ contre chez les Asiatiques ${ }^{6}$. Pourtant, le 7 octobre 2003, les Californiens allaient désavouer le projet d'amendement par $64 \%$ des voix contre $36 \%$. À travers ces chiffres, on voit bien que les électeurs californiens sont de prime abord suspicieux de cette classification. Paradoxalement, ils votèrent largement à l'inverse de leur réaction initiale.

Ce renversement de situation s'explique par la politisation du débat autour de la proposition 54. La question légitime de l'existence même des statistiques ethno-raciales et de leur rôle dans la société américaine fut bien vite écartée par les défenseurs des droits des minorités au motif que la classification ethno-raciale était nécessaire pour la recherche médicale. Loin d'être présentées comme un enjeu politique, les statistiques ethno-raciales furent présentées comme une nécessité scientifique et, partant, incontestable. L'occasion d'avoir un débat de fond sur la nature de cette classification et son rôle au sein de la société ne dura même pas le temps de cette campagne référendaire extrêmement rapide.

\section{Discours juridiques, politiques et scientifiques}

Ce qui frappe l'observateur des États-Unis est le fait qu'il existe une grande disjonction entre l'absence de races humaines prônée par la communauté scientifique et les normes sociales à travers lesquelles les populations construisent leurs vies ou leurs institutions. De la même façon, il existe une disjonction entre le caractère suspect de 
toute classification ethno-raciale dans la jurisprudence américaine, sa légitimité dans la vie des citoyens du pays et le fonctionnement des institutions. Cette classification s'est multipliée à la suite du mouvement pour les droits civiques afin de lutter efficacement contre la discrimination. Cependant, les politiques d'affirmative action, malgré une certaine popularité et des résultats tangibles, restent controversées depuis leur origine. En effet,ces politiques de promotions des minorités ethno-raciales censées corriger les pratiques discriminatoires historiques sont souvent perçues comme une entorse au principe méritocratique, même au sein des groupes pouvant potentiellement bénéficier de ces politiques. C'est pour cette raison que dans la jurisprudence des droits civiques, la classification ethno-raciale est suspecte et ne peut être tolérée que de façon temporaire et afin de remédier à un préjudice clairement identifié. La preuve doit être faite qu'une pratique discriminatoire ne peut être corrigée efficacement qu'à travers une politique publique exclusivement conçue pour répondre à un problème spécifique (narrowly tailored) et correspondant a un "intérêt gouvernemental impérieux " (compelling state interest), telle que la lutte contre la discrimination ethno-raciale. L'utilisation des statistiques ethno-raciales ne peut être justifiée que dans ce cadre précis et l'augmentation de la représentation des minorités n'est pas un but en soit, même si elle peut être justifié dans un but de diversité.

Toutefois, si le poids normatif du droit et du discours juridique est primordial aux États-Unis, pour comprendre les rouages de la rhétorique égalitaire et pourquoi elle s'articule essentiellement autour de la question de l'identité ethno-raciale, il faut admettre que cette analyse n'est pas suffisante. En effet, la mise en discours de l'égalité passe aussi par le débat public et politique qui se trouve parfois assez éloigné des considérations du juge. En effet, la justice américaine semble s'accorder sur le fait que la classification ethno-raciale est par nature suspecte et doit être soumise à un contrôle juridique fort, voire le plus strict possible (strict scrutiny). Or, on se rend très vite compte que, dans le débat public et dans le champ politique, une telle position est extrêmement minoritaire. Car il apparait que les statistiques ethno-raciales essentielles aux politiques antidiscriminatoires sont devenues l'outil essentiel sur lequel repose le discours égalitaire. La société étatsunienne semble se satisfaire de réduire la question de l'égalité à la réduction des disparités entre groupes ethno-raciaux. La lutte contre l'inégalité n'y est presque toujours mentionnée qu'en ses termes dans la mesure ou les autres formes de lutte contre les inégalités de genre, d'âge ou de classe s'inspirent en grande partie de la rhétorique de l'expérience d'émancipation des Africainsaméricains, centrale dans la construction des rapports sociaux aux États-Unis. Il semble donc que la prégnance du prisme ethno-racial ait pris le pas sur toute autre interprétation, fut elle sociale ou économique.

Pour certains, cette multiplication sans fin des statistiques dans tous les domaines de la vie des citoyens américains, même dans le but de lutter contre la discrimination, est considérée comme anathème au projet d'une société débarrassée des concepts « ethnisant » et " racisant » qui était bel et bien le projet originel du mouvement pour les droits civiques puisque ce dernier entendait débarrasser le pays de toute division raciale entre individus. Ce thème est pourtant devenu la chasse gardée des conservateurs, du moins les plus téméraires d'entre eux, ceux qui, élus dans des circonscriptions très à droite, ne craignent pas d'être taxés de vouloir maintenir la discrimination. Pour des raisons historiques et organisationnelles, le Parti démocrate a fait de la défense des politiques de traitement préférentiel des minorités un atout électoral auprès des communautés minoritaires ${ }^{7}$. C'est, partant, de la droite 
conservatrice qu'il faudra attendre une remise en question du statu quo. Mais puisque l'attaque frontale contre les politiques de traitement préférentiel de type affirmative action comporte des risques politiques lourds, les conservateurs tentent de se rabattre sur les statistiques sans lesquelles ces politiques publiques ne seraient pas possible. Et puisque le démantèlement de la classification ethno-raciale ne semble aboutir par le biais juridique c'est par le biais de la démocratie directe que les activistes tentent, dans les États qui offrent cette possibilité, de contourner le processus législatif qu'ils jugent peu propice à produire des projets de loi controversés car politiquement trop risqués.

La tentative en Californie, en 2003, d'abolir certaines formes de classifications ethnoraciales par le biais d'un référendum d'initiative populaire (Proposition 54), s'est non seulement soldé par un échec mais cet exemple illustre de façon flagrante le fossé entre la théorie juridique de justification des traitements préférentiels et le débat public. La classification ethno-raciale est perçue a priori comme le plus grand vecteur d'égalité et affirmer le contraire représente un risque politique très important pour n'importe quel élu, en particulier s'il est démocrate puisque le parti de l'âne est perçu depuis les années soixante comme le parti de l'expansion du droit des minorités et le Parti républicain a bâti sa stratégie sudiste sur cette perception. Mais au delà du clientélisme politique, la classification ethno-raciale tire sa légitimité d'un discours scientifique qui n'a jamais rompu complètement avec les préceptes du dix-neuvième siècle et cette légitimité est si forte que l'on n'hésite pas, encore aujourd'hui, à s'aventurer sur le terrain scientifique et médical pour la défendre. Rarement le lien entre discours scientifique et discours politique en ce qui concerne le concept de « race» ne fut plus évident que lors de la campagne de l'élection anticipée de 2003, où le débat sociétal tant attendu quant à l'utilité de la classification ethno-raciale n'eut jamais lieu, enseveli qu'il fut sous le poids du tabou politique et d'une ethno-racisation légitimée par le discours scientifique et médical qui réussit à faire des statistiques basées sur les concepts d'« ethnie » et de « race » des questions de vie ou de mort.

\section{La Proposition 54}

\section{Les détails du projet d'amendement}

Avant d'analyser les déboires de cet échec électoral, il convient de s'arrêter sur le texte proprement dit du projet d'amendement.

Chaque État de l'Union récolte des statistiques sur l'identité " ethnique » et " raciale » pour des raisons diverses et variées mais en général dans un but antidiscriminatoire, et souvent cette pratique est dictée par le gouvernement fédéral même si celui-ci n'a pas l'autorité pour imposer sa propre classification, c'est-à-dire celle de la directive 15 de l'office of Management and Budget ${ }^{8}$. Il tente en générale de forcer les États récalcitrant en menaçant de couper les aides financières fédérales sans nécessairement y parvenir puisque ces derniers sont très défiants vis-à-vis de leur autonomie et de leur souveraineté. Les gouvernements fédérés amassent donc des statistiques ethno-raciales sans que celles-ci ne soient ordonnées par Washington afin de mener leurs propres politiques publiques et sont très jaloux de cette prérogative ${ }^{9}$. En Californie, par exemple, l'État collecte les statistiques «ethno-raciales » des candidats dans chaque université, et ce, même s'ils ne s'inscrivent pas dans cet établissement. La Constitution de Californie permet l'utilisation de questionnaires produisant des statistiques ethno- 
raciales. Toutefois, depuis le passage de Proposition 209 en 1996, la Constitution, reprenant le vocabulaire de la loi pour les droits civiques de 1964 en en déformant le contexte, interdit, dans le domaine public, tout traitement préférentiel sur une base ethnique, raciale, de couleur de peau ou d'origine nationale.

Persuadé d'après les études d'opinion que les Californiens étaient hostiles à la classification ethno-raciale nécessaire aux statistiques, l'American Civil Rights Institute proposa un nouveau référendum d'initiative populaire. Ce dernier, la Proposition 54, aurait interdit, à partir du $1^{\mathrm{er}}$ janvier 2005, au gouvernement et à l'administration de Californie de classer les personnes suivant leur identité ethnique, raciale, leur couleur de peau ou leur origine nationale ${ }^{10}$. Cette mesure présentait, cependant, des exceptions. Premièrement, cette interdiction ne concernait pas les statistiques ordonnées par le gouvernement fédéral, ou les programmes dépendant du financement fédéral. Deuxièmement, cette interdiction ne pouvait aller à l'encontre des décisions de justice déjà en place avant le passage de l'amendement constitutionnel. Troisièmement, cette prohibition ne s'appliquait pas aux descriptions physiques des forces de l'ordre, au choix des policiers infiltrant des groupes, et à la ségrégation carcérale ${ }^{11}$. Quatrièmement, cette interdiction ne concernait pas les données statistiques nécessaires à la recherche médicale. Et cinquièmement, certaines statistiques ethnoraciales du ministère de l'emploi et du logement (Department of Fair Employment and Housing, DFEH) restaient légales jusqu'en 2014. De surcroît, ce texte permettait aussi au législateur (par un vote des deux tiers de chaque chambre), avec l'aval du gouverneur, d'approuver la collecte de statistiques ethno-raciales afin de répondre à un intérêt gouvernemental impérieux.

Il apparait donc que la grande majorité des statistiques ethno-raciales collectées par les différents services de la fonction publique de l'État de Californie n'étaient pas concernées par cette mesure puisqu'elles étaient subordonnées aux décisions du gouvernement fédéral. Seraient donc restées intactes toutes les statistiques : dans le domaine de l'emploi afin de répondre aux programmes fédéraux de lutte contre la discrimination; dans le domaine de l'éducation publique du premier et deuxième cycle en raison des examens réalisés afin d'évaluer l'écart entre les groupes ethno-raciaux ${ }^{12}$; dans le troisième cycle (University of California, California State University et community colleges) puisque ce dernier bénéficie du financement fédéral lui permettant de collecter les statistiques ethno-raciales de ses étudiants et de ses employés; dans de nombreux domaines de l'assistance publique tels que les cures de désintoxication, les hôpitaux psychiatriques ou bien les différents systèmes d'aide alimentaire aux personnes démunies (Food Stamps, etc.) dont les statistiques ethno-raciales sont ordonnées par le gouvernement fédéral ; enfin la police est aussi amenée à maintenir un système de statistiques ethno-raciales afin de répondre à différentes lois fédérales. Le DFEH étant chargé de faire respecter les lois fédérales anti-discriminatoires, une grande partie de ces statistiques ethno-raciales n'auraient pas subi de changement avec le passage de la Proposition 54. En ce qui concerne les statistiques qui n'étaient pas liées à une loi fédérale, le DFEH aurait pu les poursuivre jusqu'en 2014, date à laquelle le législateur aurait pu maintenir ces activités.

Certaines statistiques auraient toutefois été interdites par le projet d'amendement. Par exemple, toute entreprise sous contrat avec l'État n'aurait plus pu récolter de statistiques ethno-raciales ou concernant l'origine nationale. Il en allait de même pour les activités et examens des écoles publiques spécifiques à l'État de Californie ; pour les 
candidats (non inscrits) dans les University of California et les California State Universty; les élèves de lycée participant à des activités organisées par les UCs; les étudiant de troisième cycle participant au programme d'effacement de leur prêt étudiant ; les étudiants passant les concours d'enseignement (teaching credentials).

Enfin, de par la formulation du texte, les effets concernant certains dispositifs restaient flous avec leur application dépendant de l'interprétation de l'amendement par la justice et le législateur. Par exemple, les données démographiques de l'État de Californie ne se basent pas que sur les résultats du recensement. L'État de Californie utilise les estimations fournies par l'Unité de recherche démographique (Demographic Research Unit ou DRU) du ministère des Finances de l'État. De plus, une fois compilées, les données du recensement (et toutes les autres statistiques récoltées afin de répondre aux exigences fédérales) servent à de nombreuses politiques publiques. Il n'était pas clair dans quelle mesure et dans quel but les données démographiques auraient pu être utilisées. Par exemple, les criminologues se demandaient si, bien que la police ait eu le droit de décrire physiquement des suspects, les taux de criminalité par groupe ethnoracial auraient été maintenus. Mais, le domaine le plus controversé, resta celui de la recherche médicale. Concernant cette exception, une très grande majorité de professionnels de santé ne furent pas convaincus que le texte leur garantissait le maintien des différentes applications de la classification en médecine. Le texte semblait assez vague pour pouvoir être interprété ultérieurement par la justice ou le législateur comme interdisant la collecte des statistiques dans certains domaines utiles ou pouvant se révéler cruciaux pour des questions de santé publique. Comment, par exemple, suivre l'évolution du taux de diabète des enfants hispaniques si de telles statistiques étaient interdites dans les hôpitaux publics sous prétexte que l'État ne peut participer à la classification ethno-raciale de sa population?

Ces arguments contre la Proposition 54 furent exprimés et résumés dans le guide officiel des électeurs de Californie qui, pour chaque Proposition soumise au jugement des électeurs,présente les opinions des deux camps ${ }^{13}$. Dans un texte rédigé par Jacqueline Jacobberger (présidente de la ligue des électrices de Californie déjà très impliquée dans la lutte contre la Proposition 209), John C. Lewin (président de l'Association médicale de Californie), et Robert M. Pearl (du géant de l'assurance maladie Kaiser Permanente), les détracteurs de la Proposition 54 expliquent que cet amendement proposé à la Constitution de l'État représente une atteinte au droit à l'information. Cette classification ethno-raciale est une source d'information primordiale dans le domaine du suivi des maladies infectieuses par exemple. Les auteurs expliquèrent donc que « si une épidémie se déclarait dans un groupe, nous devrions pouvoir l'identifier et la contenir avant qu'elle ne s'étendît à la population générale » ${ }^{14}$. Ce point, qui ne fut soulevé par aucun intellectuel de renom ou aucun media de masse, rappelle étrangement le vieux mythe xénophobe des groupes ethno-raciaux qui contamineraient la population de leurs maladies contagieuses ${ }^{15}$. Par conséquent, suivant cet argument, une maladie se déclarant aux États-Unis ne pourrait pas être enrayée sans classer la population en groupes ethno-raciaux ${ }^{16}$. Une telle situation de ségrégation épidémiologique semble pourtant bien improbable ${ }^{17}$.

Les détracteurs de la Proposition 54 enfoncent le clou en précisant que «l'exception médicale " contenue dans le projet d'amendement permet seulement de classer les patients individuellement et ne permet pas de classer « les populations afin de prévenir les maladies", et citent des organisations médicales prestigieuses opposées à la 
Proposition (telles que l'Académie des médecins de famille de Californie (California Academy of Family Physicians), l'Académie américaine de pédiatrie (American Academy of Pediatrics), l'Association de Californie de la santé (California Healthcare Association), ou l'Association californienne des hôpitaux publics (California Association of Public Hospitals).

Il n'en demeure pas moins que la l'utilité de telles statistiques face à la prévention des maladies reste contestée dans d'autres milieux scientifiques ${ }^{18}$. L'opposition s'appuie ainsi sur les travaux de deux généticiens, Susanne Haga et J. Craig Venter, pour déclarer qu'appliquer des catégories "dépassées» à l'interprétation des données scientifiques pourrait mener à des conclusion erronées et biologiquement insignifiantes. L'équipe de Jaccobberger affirme pourtant que «les femmes blanches ont un taux de cancer du sein supérieur " (sans préciser par rapport à qui), "les Asiatique-américains ont un risque plus grand de contracter l'hépatite B » (sans préciser par rapport à qui), "les Hispaniques sont plus susceptibles de mourir des causes liées au diabète » et les "Afro-américains ont un plus fort taux de décès dû aux maladies cardiaques» (toujours sans préciser par rapport à qui) ${ }^{19}$. Et de conclure : "cette information [obtenue par la classification ethno-raciale] sauve des vies". L'argument est de taille. Et pour appuyer cette conclusion, les opposants à la Proposition 54 rappellent que plus de 40 autres groupes se joignent à eux telles l'Association américaine des maladies du cœur (American Heart Association), Action contre le cancer du Sein (Breast Cancer Action), ou encore la Coalition hispanique pour une Californie en bonne santé (Latino Coalition for a Healthy California).

L'argument de Ward Connerly en faveur de la Proposition 54 est qu'il est primordial de se souvenir de l'origine raciste de cette forme de classification et d'apprendre des erreurs de l'histoire, de l'eugénisme et du national socialisme. De plus, d'après lui, la discussion autour de la multiracialité a démontré que l'idée de races pures et hermétiques est caduque $^{20}$. Mais ces arguments firent pâle figure à côté de cette déferlante scientifique. De plus, l'équipe de Jacobberger put s'enorgueillir de bénéficier du soutien d'autres associations judiciaires ou policières qui estimèrent que le texte de la Proposition laissait la porte ouverte à un démantèlement du système judiciaire ${ }^{21}$. Les rues seraient donc moins sûres si la justice ne disposait pas de statistiques criminelles classées par groupe ethno-racial. Le milieu éducatif se sentit aussi menacé et apporta sa pièce à l'édifice (grâce notamment à ses puissants syndicats) et Jacobberger et ses collègues expliquèrent que le California Public School Accountabily Act fut mis en place pour s'assurer que les écoles réduisaient l'écart scolaire (achievement gap) entre les différents groupes ethno-raciaux et que le passage de la Proposition 54 ne permettrait pas de vérifier que les établissements scolaires feraient correctement leur travail ${ }^{22}$.

Les électeurs devaient donc choisir entre deux textes radicalement opposés. D'un côté, un projet d'amendement qui dénonçait la classification ethno-raciale révolue et héritée d'un autre temps, ainsi que l'immixtion du gouvernement dans le domaine de l'identité personnelle et intime de chacun. Mais, ne pouvant poursuivre ce raisonnement jusqu'au bout, les auteurs de la Proposition 54 y inclurent de nombreuses exceptions plus ou moins claires. De l'autre côté, une coalition très vaste de représentants d'associations, de syndicats et de corporations professionnelles respectées des électeurs : la justice, l'éducation et la santé. Le passage de la Proposition 54 équivaudrait à une censure de l'information. Une information nécessaire et qui sauve des vies. 


\section{La nature des débats référendaires}

Il faut signaler à ce moment de notre étude que les arguments résumés de façon succincte par les deux camps et présentés aux Californiens dans le guide électoral ne peuvent tenir lieu de réel débat sociétal. Et ce pour plusieurs raisons qui ont trait à la nature même des référendums d'initiative populaire. Premièrement, peu d'électeurs parcourent ces guides qui sont souvent épais puisque le nombre de référendums, locaux notamment, semble augmenter à chaque élection ou presque. Deuxièmement, ces argumentaires sont écrits par des groupes politiques peu objectifs, voire non indépendants, dont le rôle n'est pas d'éduquer le lecteur mais bien de le convaincre. Troisièmement, et c'est peut-être là la plus grande faiblesse du système référendaire tel qu'il se présente en Californie, les textes de référendums sont presque toujours obscurs, opaques voir incompréhensibles. La genèse tortueuse de ces textes qui sont le plus souvent le résultat de circonstances politiques fait qu'ils sont très peu propices à stimuler le débat démocratique. Soit ils sont écrits de façon trop rapide pour répondre à un impératif politique, ils sont confus et prêtent parfois le flanc au recours en justice. Soit ils sont écrits avec soin par des spécialistes du droit mais dans ce cas il n'est pas rare qu'ils soient formulés de façon à occulter le but réel de leurs auteurs. Car dans la majorité des cas le but du référendum est bien de "court-circuiter» le système législatif. En effet, pour des sujets parfois techniques et souvent complexes, les auteurs tentent souvent de camoufler la controverse qu'ils pourraient susciter dans un jargon juridique et législatif qui rend le texte abscond. Tant et si bien que même les professionnels de la politique californienne doivent eux-mêmes souvent s'en remettre à leurs collègues lobbyistes ou attachés parlementaires spécialistes de la question afin d'entrevoir les possibles conséquences de chaque amendement constitutionnel. C'est pour cette raison aussi que l'argumentaire est si important puisque de nombreux électeurs se déterminent simplement grâce au nom des organisations soutenant ou opposant le projet. En fait, les référendums d'initiative populaire sont par nature de très mauvais vecteurs de débat politique de fond ${ }^{23}$.

Enfin, avant de revenir à la dynamique politique qui caractérisa cette élection, il nous faut rappeler que la chute spectaculaire du projet d'amendement dans les sondages et sa défaite finale furent aussi déterminées en partie par le contexte de cette élection exceptionnelle à bien des égards.

\section{Le contexte de l'élection}

Dans l'espace de quelques mois la tendance s'inversa complètement vis-à-vis de la Proposition 54. Comment expliquer un tel revirement de situation? Il faut avoir à l'esprit tout d'abord le contexte complexe de cette élection exceptionnelle. Rappelons que le système de référendum d'initiative populaire californien, dans lequel l'argent joue un rôle primordial notamment dans la récolte des signatures, permit de destituer, lors de la même élection, un gouverneur, par la voie démocratique, onze mois seulement après que celui-ci ait été réélu. Darrell Issa, riche homme d'affaire et membre de la chambre des représentants du Congrès pour la très conservatrice 49ème circonscription de Californie, lança sa campagne de révocation du gouverneur Davis en payant des «suburban warriors $»^{24}$, ces partisans fidèles que la droite américaine sait si bien mobiliser en Californie du Sud, afin de collecter le million de signatures nécessaires 
pour lancer le référendum, pensant qu'il serait candidat si le processus aboutissait. Hélas, ce dernier lui échappa et il fut vite écarté de la campagne par les mastodontes du Parti républicain, surtout lorsque Arnold Schwarzenegger et Tom McClintock se portèrent candidats. Le camp démocrate tenta d'enrailler le vote de destitution mais après moult péripéties judiciaires le référendum fut avalisé par la justice et la Proposition 54, initialement prévue pour les primaires de mars 2004, se retrouva projetée dans cette arène électorale qui prit un peu les Californiens au dépourvu. Le projet d'amendement de la Constitution fut donc pris au milieu d'une élection du gouverneur à quelques 135 candidats et une campagne éclair de deux mois. Ajoutons à cela une controverse concernant les bulletins de vote qui étaient les mêmes que ceux utilisés lors du fiasco floridien de l'élection présidentielle de $2000^{25}$, et on comprend vite que cette cacophonie électorale n'était pas propice à créer les conditions sereines d'un débat de fond ${ }^{26}$.

Ensuite, et c'est un point essentiel, la Proposition 54 est arrivée sur l'échiquier politique au moment de l'élection de destitution, ce qui avait pour conséquence de jeter de l'ombre sur le débat de fond ${ }^{27}$. En effet, toute campagne électorale américaine est en générale déjà marquée par des enjeux ethno-raciaux ou communautaires. Puisque les minorités votent traditionnellement démocrate, l'enjeu électoral visant à courtiser les électeurs noirs et surtout hispaniques dans le cas de la Californie, est de taille ${ }^{28}$. Gray Davis, dans un ultime fait d'armes, s'empressa de signer un projet de loi visant à permettre aux sans-papiers californiens d'obtenir un permis de conduire, espérant ainsi recueillir quelques électeurs de dernière minutes dans la communauté hispanique, celle-ci comptant en son sein la très grande majorité des sans-papiers ${ }^{29}$. Cette décision eut pour effet de relancer le débat sur l'immigration illégale, question à connotation ethno-raciale puisque l'immigration illégale est perçue comme un problème hispanique ${ }^{30}$. De plus, une autre source de controverse émana de la présidente de la branche californienne du NAACP, Alice Huffman, qui accusa Schwarzenegger d'être raciste et d'avoir été influencé par l'appartenance de son père au Parti nazi en Autriche ${ }^{31}$. Il faut aussi ajouter à tout cela le scandale du financement de la campagne de Cruz Bustamante, le gouverneur adjoint, accusé d'avoir bénéficié de façon illégale de contributions très importantes de tribus amérindiennes ${ }^{32}$. On le voit bien, la politique californienne regorge de considérations communautaires et les participants connaissent le pouvoir mobilisateur de thèmes aussi passionnels. La Proposition 54, qui visait à éliminer certaines formes de classification ethno-raciale émanant des pouvoirs publics, envenima la situation et allait se retrouver elle-même prisonnière des conditions politiques du moment.

L'American Civil Rights Institute est une organisation conservatrice très proche du Parti républicain au sein duquel elle compte ses principaux donateurs. Le niveau d'antipathie vis-à-vis de Gray Davis et ses scandales financiers de gestion de l'argent public, était si élevé que le Parti républicain voyait dans cette élection une opportunité de reprendre la direction de l'État le plus peuplé et le plus riche de l'Union, mais aussi un État dont la branche législative est traditionnellement contrôlée par les démocrates. Or, la Proposition 54 de l'ACRI était tout à fait le genre de controverse dont le camp républicain se serait passé. Très vite, les analystes politiques ont supposé que la Proposition 54 était susceptible de motiver de nombreuses personnes issues des groupes minoritaires à aller aux urnes, ce qui faisait le jeu de Davis, qui avait été réélu in extremis en 2002 grâce notamment à l'apport des voix des Africains-américains et des Hispaniques ${ }^{33}$. Le Parti républicain, qui dans son ensemble n'était déjà pas très favorable à la Proposition $54^{34}$, 
prit très vite ses distances avec Ward Connerly qui se retrouva esseulé face à un Parti démocrate et une opposition très bien organisée (et financée), et ayant tiré les enseignements de son échec lors de la campagne sur la Proposition 209. La campagne en faveur de la Proposition 54 devait lutter contre les attaques de Cruz Bustamante, le gouverneur adjoint, candidat démocrate à la succession de Gray Davis, qui la prit pour cible à travers une lourde campagne télévisuelle espérant ainsi motiver la base de son parti. Et par conséquent, le référendum devait aussi lutter contre les pressions politiques du camp de Schwarzenegger qui voyait dans la Proposition un obstacle à son élection puisque susceptible de mobiliser l'adversaire ${ }^{35}$. Car sans le soutien unanime du Parti conservateur, ses chances de gagner l'élection s'étaient considérablement amoindries $^{36}$. Toutefois, ce contexte n'explique qu'en partie le sort de la Proposition 54. Car s'il offre un début d'explication quant à la défaite dans les urnes, il n'explique pas pourquoi les questions légitimes soulevées par ce projet d'amendement, à savoir le rôle de la classification ethno-raciale dans la société américaine, allaient être complètement et définitivement passées sous silence. Au-delà du caractère chaotique de l'élection et des entrecroisements des intérêts de partis et des candidats, il est essentiel de noter qu'il n'y eut même pas un semblant de débat sur le thème de cette classification. Ce "silence assourdissant» est si remarquable que nous nous devons d'en chercher les implications et les explications.

\section{L'absence de débat public sur la classification ethno- raciale et le rôle du Parti démocrate}

L'épisode californien nous en dit long sur la conceptualisation de la notion de race aux États-Unis et sur les limites du débat public et politique quant à l'instrumentalisation de la classification ethno-raciale. En fait, de débat il ne fut jamais vraiment question. Aucun journaliste, homme politique ou intellectuel de renom ne sembla vouloir considérer la possibilité que l'utilisation de la classification n'était pas la pierre angulaire de la justice et de l'égalité dans le système démocratique américain. Les hommes politiques, républicains et démocrates, se sont vite rendu compte qu'ils risquaient de se mettre à dos une grande partie de l'opinion publique s'ils remettaient en cause ce marqueur identitaire très fort que représentent les statistiques ethnoraciales. Car la Proposition 54 se vit très rapidement taxée de "prohibition de l'information " (Information Ban). Pourtant, très peu d'universitaires ou d'intellectuels crurent bon d'intervenir afin de demander de quel genre d'information il était question. L'information était-elle de nature biologique ? Ou bien l'information était-elle de nature sociologique avec des implications biologiques? Cette question est primordiale et est au cœur du débat scientifique aux États-Unis, comme l'illustrent les discussions sur l'émergence de la pharmacogénétique et de l'identification ethnoraciale par l'ADN ${ }^{37}$. Les scientifiques américains ne semblent pas avoir atteint un consensus sur la nature même du concept de race et un débat approfondi s'impose afin de déterminer pour quelles raisons la classification ethno-raciale doit être maintenue, si tant est qu'elle le doive ${ }^{38}$.

Ce débat public pourtant légitime n'eut pas lieu car cette question personne ne la posa et la Proposition 54 disparut très vite sous le rouleau compresseur de l'argument médical qui avançait que l'absence de classification empêchait les chercheurs d'étudier les maladies et les médecins de guérir les Californiens. Dans un spot publicitaire, Everett C. 
Koop, ancien ministre de la santé (Surgeon General of the United States), déclara, sans sourciller, que la classification ethno-raciale était « une affaire de vie ou de mort »! Dès lors, même s'ils avaient été avancés, les arguments épistémologiques ou philosophiques sur la nature du concept de race n'auraient surement pas suffit à lancer le débat si le public était persuadé que de telles statistiques permettaient de sauver des vies. Le gouverneur adjoint Cruz Bustamante, candidat institutionnel du Parti démocrate, allait même voir dans la Proposition 54, et l'engouement qu'elle pouvait susciter, un support pour sa campagne ${ }^{39}$. Il fut accusé notamment de contourner la loi sur le financement des campagnes électorales ${ }^{40}$ en bénéficiant de très grosses sommes d'argent provenant des groupes de pression amérindiens ${ }^{41}$, enrichis par l'industrie des casinos, à travers un organisme censé combattre la Proposition $54^{42}$. Fort de ces quelques 4 millions de dollars supplémentaires, il lança une série de publicités télévisées ${ }^{43}$, très diffusées, affirmant que la classification ethno-raciale était source d'information médicale et sauvait des vies, en particulier celles des minorités qui sont plus susceptibles d'être en mauvaise santé, sous-entendant ainsi que la santé publique était un sujet plus important pour les minorités ${ }^{44}$. Afin de lutter contre cette déferlante médiatique Ward Connerly fit lui aussi appel à ses relations pour financer sa campagne de façon plutôt opaque ${ }^{45}$.

De nombreux universitaires et chercheurs savent pertinemment que bien des pays de par le monde n'ont pas recours à la classification ethno-raciale et que l'on n'y est pas forcément plus mal soigné. Les États-Unis dépensent pour leur système de santé deux fois plus que les autres pays industrialisés pour des résultats plus médiocres, comme en atteste entre autres l'espérance de vie ou le taux de mortalité infantile ${ }^{46}$. Ils savent qu'un système de couverture maladie universelle gommerait une grande partie des disparités de santé entre les différents groupes sociaux et c'est une des raisons qui a poussé le président Obama à passer sa réforme de l'assurance maladie en $2010^{47}$. Pourtant, les seuls médecins qui prirent la parole confirmèrent l'argument médical en faveur de la classification ethno-raciale. Les historiens des sciences, les sociologues et autres intellectuels restèrent tellement discrets que l'on peut se demander s'ils n'acquiesçaient pas par leur silence. C'est que le Parti démocrate et les associations progressistes proches du parti firent de la Proposition 54 leur étendard contre le retour des conservateurs, de Ward Connerly et du fantôme de la défaite face à la Proposition 209. La Proposition 54 fut habilement liée par le camp progressiste au vote de destitution du gouverneur démocrate puisqu'il se déroulait en même temps. Ces deux événements allaient être présentés inlassablement comme deux attaques simultanées et combinées de la droite conservatrice: l'une contre le Parti démocrate, l'autre contre les minorités ${ }^{48}$. Le Parti démocrate tenta ainsi de renforcer un peu plus l'adage selon lequel il est l'allié des minorités. Toute personne suggérant que la Proposition 54 ne représentait pas nécessairement la fin de la médecine moderne se retrouvait isolée et risquait de se voir taxée au mieux de conservateur, au pire de raciste. Et apparaître comme raciste, ou au moins ne pas être clairement en faveur des minorités ethnoraciales, est très souvent pour les élus démocrates perçu comme un véritable risque politique. Seuls certains républicains peuvent se risquer à s'opposer aux intérêts des groupes minoritaires sans perdre tout crédit vis-à-vis de leur électorat. Toutefois, si Connerly est financé et instrumentalisé par les conservateurs, il n'en demeure pas moins que sa question était d'un point de vue intellectuel on ne peut plus légitime. Audelà des clivages politiques, la Proposition 54 présentait à la société américaine une question essentielle qui ne fut finalement pas posée. Les porte-paroles de la gauche américaine se sont arc-boutés, régurgitant l'argument médical sans en digérer les 
implications épistémologiques. L'utilisation des statistiques ethno-raciales dans le domaine de la santé publique et de la médecine leurs octroie une légitimité scientifique, qu'elles n'ont d'ailleurs jamais vraiment perdu aux États-Unis, et de laquelle résulte le droit d'intervenir sur l'homme non plus à partir de ce qu'il est par statut mais de ce qu'il est par nature, selon sa constitution. Suggérer que les statistiques ethno-raciales dans les études médicales ne seraient qu'une donnée démographique, au même tire que l'âge ou le sexe, fait courir le risque de réifier le concept de races humaines en le biologisant.

Par exemple, on pouvait apprendre dans les pamphlets du très actif Syndicat américain des libertés civils (ACLU), association progressiste qui fait par ailleurs un travail remarquable: "Le cancer du sein, les problèmes cardiaques, la mortalité infantile, l'anémie falciforme, le cancer de la prostate, le Sida, la tuberculose et le cancer des cervicales sont seulement quelques-unes des maladies qui nous affligent différemment en fonction de notre race $»^{49}$. L'identité ethno-raciale n'est plus qu'une vérité médicale avérée, un concept scientifique sans équivoque ni controverse. Ces arguments allaient être repris par la presse, notamment le San Francisco Chronicle, qui prit officiellement position contre la Proposition 54 en ces termes :

Pire, la Proposition 54 interdit la collecte d'informations qui sont essentielles pour la santé publique. Elle contient une exception très étroite pour «les patients et les sujets de la recherche médicale " mais elle interdirait la prise en compte de l'identité raciale sur les certificats de naissance et de décès, ainsi que d'autres données démographiques vitales pour les organismes de santé publique.

L'ignorance est la mère de tous les vices. Votez non à la Proposition $54^{50}$.

Refuser de classer les être humains sur une base « ethnique » ou « raciale » serait donc source d'ignorance ${ }^{51}$. L'identité ethno-raciale représente une source d'information. Pourtant, si ce présupposé est vrai, personne ne prit le temps de définir de quel genre d'information il s'agissait. Une information de nature génétique? Ou bien une information de nature sociologique? Ou bien une information d'une toute autre nature? Il semble que la conception américaine de la médecine ne fasse pas la part belle à la sociologie. Les considérations génétiques et "biologisantes " y sont omniprésentes et un débat sur la nature de l'information créée par la classification ethno-raciale aurait pu, aurait dû, clarifier ou du moins amener sur le devant de la scène politique et du débat public cette interrogation primordiale.

\section{Conclusion : rôle du discours scientifique dans le débat politique et identitaire}

Les données médicales, et à travers elles les statistiques ethno-raciales, sont perçues comme des données de nature biologique, forcément objectives et, partant, incontestables. Cependant, la classification ethno-raciale est avant tout une considération d'ordre politique. Ne pouvant se résoudre à remettre en cause cette classification, ou du moins à entamer une réflexion sur les implications philosophiques mais aussi pratiques de cette dernière, l'opinion américaine et la classe politique se sont contentées d'instrumentaliser le discours scientifique ambiant afin de maintenir cette perspective. La société étatsunienne semble entretenir, à travers cette essentialisation de la médecine, une certaine " désociologisation » de l'identitaire en ce qui concerne le concept de race. A travers la Proposition 54, comme à travers la commercialisation de médicaments « ethniques » tels que le Bidil ${ }^{52}$, on peut observer le 
rôle du discours scientifique et ses points d'ancrage profonds sur la pensée identitaire et sur l'organisation politique de la société états-unienne. Ce silence rédhibitoire face aux questions soulevées par le référendum d'initiative populaire illustre les limites d'une autocritique sociologique états-unienne face au besoin presque irrépressible de légitimation d'une classification ethno-raciale sans laquelle il n'existe pas de progrès scientifique, d'accès au savoir et, surtout, de socle identitaire. Les Californiens virent dans la Proposition 54 une atteinte à leurs identités personnelles et leur sens identitaire collectif, menacé de disparaître. Car cette classification semble bel et bien représenter pour les Américains une voie privilégiée de la connaissance de soi et de l'Autre.

\section{BIBLIOGRAPHIE}

BENNER, Laurence A, «Prop. 54 ; A dangerous veil of ignorance », San Diego-Union Tribune, 1 octobre 2003.

BERNARD, Philippe \& WEIL, Nicolas, « Une virulente polémique sur les données « ethniques » divise les démographes », Le Monde, 6 novembre 1998, 10.

BERTHELSEN, Christian, « Bustamante told not to use loophole », San Francisco Chronicle, 23 septembre 2003, A1.

«Prop. 54's big-money backers revealed », San Francisco Chronicle, 19 mai 2005, B2.

CAHN, Steven M. (ed.), The Affirmative Action Debate, New York \& London, Routledge, 2002.

COILE, Zachary, « Tribes add millions to McClintock, Bustamante », San Francisco Chronicle, 27 septembre 2003, A18.

«Question on NAACP boss' racism charge », San Francisco Chronicle, 3 octobre 2003, A14.

CONNERLY, Ward, Creating Equal: My Fight Against Race Preferences, San Francisco, CA, Encounter Books, 2000.

Del OLMO, Franck, « Now Latinos Are in the Driver's Seat», Los Angeles Times, 27 juillet 2003, M5.

Editorial, « The Recall Reargued », New York Times, 23 septembre 2003, A30.

Editorial, « The San Francisco Chronicles Recommends/ PROP 54, Racial data: No »,San Francisco Chronicle, 5 octobre 2003, E8.

FEARS, Darryl, « Calif. Activist Seeks End To Identification by Race », Washington Post, 5 juillet 2003, A1.

FREEMAN, Jo, « The Political Culture of the Democratic Party and the Republican Party », Political Science Quarterly, vol. 101, n³, 1986, 327-56.

HADDOCK, Vicky, « Politcs and Race », San Francisco Chronicle, 28 septembre 2003, D3.

HALL, Prescott F, Immigration and its Effects upon the United States, New York, NY, Henry Holt, 1906.

HENDRICKS, Tyche, «Latinos Activists gear up to register, get out vote », San Francisco Chronicle, 13 août 2003, A16. 
KRIEGER, Nancy, « Does Racism Harm Health? Did Child Abuse Exist Before 1962? On Explicit Questions, Critical Science, and Current Controversies: An Ecosocial Perspective », American Journal of Public Health, février 2003, vol. 93, n², 194-99.

« A Glossary for social epidemiology », Journal of Epidemiology and Community Health, October 2001, vol. 55, 693-700.

« Discrimination and Health », in BERKAMN Lisa F. et KAWACHI Ichiro (dir.), Social Epidemiology, Oxford: Oxford University Press, 2000, 36-75.

LeDUFF, Charlie, « Ethnic Issue in California Recall Play Out at Latino Parade », New York Times, 8 septembre 2003, A19.

LONG, Emmet (ed.), Affirmative Action, New York, NY, The H.W. Wilson Company, 1996.

MAGAGNINI, Stephen, « Poll bucks prior surveys, sees Prop. 54 winning », Sacramento Bee, 26 septembre 2003, A3.

McGRIRR, Lisa, Suburban Warriors: The Origins of the American Right, Princeton University Press, 2002.

MORAIN, Dan \& RUBIN, Joel, « Bustamante Ads Still on the Air », Los Angeles Times, 25 septembre 2003, A26.

MORNING, Ann. 2009, «Toward a Sociology of Racial Conceptualization for the 21st Century ", Social Forces 87(3), 1167-92.

MURPHY, Dean E, « Affirmative Action Foe's Latest Effort Complicates California Recall », New York Times, 3 août 2003, 1.13.

«California's Vote Delayed by Court over Punch Cards », New York Times, 16 septembre 2003, A1.

NELSON, Jennifer, "A Reluctant No On 54: We Need The Boxes ", San Francisco Gate, 8 septembre 2003, Disponible sur : http://www.sfgate.com/cgi-bin/article.cgi ?file =/gate/archive/ 2003/09/08/jnelson.DTL (page consultée le 23 avril 2009).

POST, Robert \& ROGIN, Michael (eds.), Race and Representation: Affirmative Action. New York, NY, Zone Books, 1998.

RAHEMTULLA, Talsin \& BHOPA, Raj, «Pharmacogenetics and ethnically targeted therapies: New drug BiDil marks the return of biology to the debate aboutrace and ethnicity ", British Medical Journal, vol. 330, 7 mai 2005, 1036-37.

RAINE, George, « Bustamante airs first anti-Prop. 54 ad », San Francisco Chronicle, 18 septembre 2003, A12.

RICHOMME, Olivier, « La classification « ethno-raciale » des statistiques démographiques aux États-Unis », Annales de démographie historique, 2007, 꾸1, 177-202.

ROJAS, Aurelio, « Bustamante to shift disputed donations The \$3.8 million move to fight Prop. 54 aims to quiet his critics ", Sacramento Bee, 7 septembre 2003, A1.

SAMPLE, Herbert A, « Jesse Jackson fights recall and Prop. 54», Sacramento Bee, 17 septembre 2003, A3.

SAVAGE, David G. \& Warren, Jenifer, « Justices Reject Segregation in State's Prisons », Los Angeles Times, 24 février 2005, A1.

SCHEVITZ, Tanya, « Recall's Strange Bedfellow », San Francisco Chronicle, 29 juillet 2003, A11. 
SCHRAG, Peter, « Race and gubernatorial recall: Can they be linked?», Sacramento Bee, 30 juillet 2003, B7.

« In California, White Men Are the Silent Plurality », New York Times, 14 septembre 2003, 3.

SIMON, Mark, « Indian campaign donations in the spotlight », San Francisco Chronicle, 24 septembre 2003, A18.

SPICKARD, Paul R, «The Illogic of American Racial Categories », in Maria P. ROOT, Racially Mixed People in America, Newbury Park, London, New Delhi, Sage Publications, 1992.

STEEL, Shelby, The Content of Our Character, New York, NY, Harper Perenial, 1991.

TROUNSON, Rebecca, HELFAND, Duke \& BUSTILLO, Miguel, « Racial Data Measure May Be a Wild Card in Election ", Los Angeles Times, 15 août 2003, A30.

U.S. CENSUS, «Income, Poverty, and Health Insurance Coverage in the United States: 2007», 2008, http://www.census.gov/prod/2008pubs/p60-235.pdf (page consultée le 24 mai 2011).

U.S. OFFICE OF MANAGEMENT AND BUDGET, « Directive n¹5, Race and Ethnic Standards for Federal Statistics and Administrative Reporting », Federal Regulations, 1978, vol. 43, n¹9, 260.

WADE, Nicholas, « Race Is Seen as Real Guide To Track Roots of Disease», New York Times, 30 juillet 2002, F1.

« For Sale: A DNA Test To Measure Racial Mix », New York Times, 1er octobre 2002, F.4.

«Scientists differ on genetics of race », International Herald Tribune, 28 octobre 2004, 6.

« Race-Based Medicine Continued... », New York Times, 14 novembre 2004, 4.12.

WALTER, Dan, « Tribes lose high-stakes political gamble in recall election », Sacramento Bee, 20 octobre 2003, A3.

WEBBER Henry S., « The Failure of Health Care Reform: An Essay Review », The Social Service Review, vol. 69, n², juin 1995, 318.

WILLIAMS David R., LAVIZZO-MOUREY Risa et WARREN, Rueben C. « The Concept of Race and Health Status in America », Public Health Report, janvier/février 1994, vol. 109, 26-41.

ZACK, Naomi, Philosophy of Science and Race, New York \& London, Routledge, 2002.

\section{NOTES}

1. LONG, Emmet (ed.), Affirmative Action, New York, The H.W. Wilson Company, 1996; POST Robert \& ROGIN, Michael (eds.), Race and Representation: Affirmative Action, New York, Zone Books, 1998; CAHN, Steven M (ed.), The Affirmative Action Debate, New York \& London, Routledge, 2002.

2. Les critiques se limitent souvent à remettre en question les politiques publiques et non la classification en elle-même. STEEL, Shelby, The Content of Our Character, New York, Harper Perenial, 1991 ; Ceux qui attaquent la classification directement perdent souvent leur légitimité politique en étant trop ouvertement instrumentalisés par les conservateurs. CONNERLY, Ward, Creating Equal: My Fight Against Race Preferences, San Francisco, Encounter Books, 2000.

3. FEARS, Darryl, «Calif. Activist Seeks End To Identification by Race », Washington Post, 5 juillet 2003, A1.

4. McGRIRR, Lisa, Suburban Warriors: The Origins of the American Right, Princeton University Press, 2002. 
5. MAGAGNINI, Stephen, «Poll bucks prior surveys, sees Prop. 54 winning », Sacramento Bee, 26 septembre 2003, A3. Ce sondage fut effectué dans six langues.

6. Notons tout de même un bémol à cette étude car $44 \%$ des Blancs étaient encore indécis.

7. Sur la structure et la culture de coalition du Parti démocrate basé sur les caucus cf. par exemple FREEMAN, Jo, "The Political Culture of the Democratic Party and the Republican Party ", Political Science Quarterly, vol. 101, n³ 1986, 327-56.

8. Cette directive, adoptée par le Ministère du commerce dont dépend le Bureau du recensement, est devenue la norme fédérale en matière de catégorisation ethno-raciale. U.S. OFFICE OF MANAGEMENT AND BUDGET, « Directive nำ15, Race and Ethnic Standards for Federal Statistics and Administrative Reporting », Federal Regulations, 1978, vol. 43, n¹9, 260.

9. Pour une discussion plus approfondi du système de statistiques ethno-raciales de l'État de Californie cf. RICHOMME, Olivier, «La classification «ethno-raciale» des statistiques démographiques aux États-Unis », Annales de démographie historique, 2007, no1, 177-202.

10. La question de l'origine nationale est parfois assimilée à la classification ethno-raciale alors qu'elle ne soulève pas les mêmes questions épistémologiques. De plus, l'origine nationale ne fait pas réellement partie de la controverse car aux États-Unis c'est à travers le prisme du pentagone ethno-racial qu'est envisagée la question de l'Autre.

11. Entre temps, la Cour suprême a déclaré que la ségrégation carcérale était difficilement justifiable aux yeux de la Constitution au grand dam des syndicats de gardiens et de responsables de l'administration pénitentiaire qui voient la ségrégation comme le seul moyen de garantir la sécurité des prisonniers. Aux États-Unis, de nombreux gangs s'organisent autour de l'identité communautaire, géographique et ethno-raciale. SAVAGE, David G. \& Warren, Jenifer, « Justices Reject Segregation in State's Prisons ", Los Angeles Times, 24 février 2005, A1.

12. Aux États-Unis, les familles remplissent des fiches de renseignement en début de chaque année scolaire (dès le plus jeune âge) sur lesquels figure l'identité ethno-raciale des enfants. Généralement remplies par les parents, il est possible qu'elles soient remplies par l'enseignant. Il semble aussi qu'une fois arrivés au lycée les élèves puissent remplir leur fiche de renseignement tout seul. Ces données sont une part essentielle de la politique « No Child Left Behind » lancée par le président Bush qui vise, entre autres choses, à travers des examens nationaux standardisés, à évaluer l'écart entre les groupes ethno-raciaux dans le but, évidemment, de le réduire.

13. CALIFORNIA STATEWIDE SPECIAL ELECTION, Tuesday October 7th 2003, Official Voter Information Guide,Possession de l'auteur.

14. Ibid.

15. Ce thème est notamment très présent dans les arguments de justification des restrictions à l'immigration de 1921, 1924 et 1929 ainsi que dans le discours eugéniste qui l'accompagnait. Cf. par exemple HALL, Prescott F, Immigration and its Effects upon the United States, New York, Henry Holt, 1906.

16. La classification d'après l'origine nationale soulève moins de controverse dans l'épidémiologie même si elle peut s'avérer aussi réductrice.

17. Cf. notamment les travaux de Nancy Krieger qui en appelle à une perspective " écosociale » du racisme, ou une «épidémiologie sociale ». Expression révélatrice puisqu'elle sous entend que certains épidémiologistes américains envisagent leur discipline comme dépourvue de toute considération sociale. La conséquence directe de ce manque de contextualisation sociale est la profusion de travaux réduisant l'environnement à une catégorie socioprofessionnelle dans lesquels il n'est pas question d'observer les «expressions biologiques de la racisation ou du sexage ». KRIEGER, Nancy, « Does Racism Harm Health? Did Child Abuse Exist Before 1962? On Explicit Questions, Critical Science, and Current Controversies: An Ecosocial Perspective ", American Journal of Public Health, février 2003, vol.93, n², 194-99; «A Glossary for social epidemiology ", Journal of Epidemiology and Community Health, October 2001, vol.55,693-700; 
« Discrimination and Health », in BERKAMN, Lisa F. et KAWACHI, Ichiro (dir.). Social Epidemiology, Oxford, Oxford University Press, 2000, 36-75.

18. On observe, pour certaines maladies, des probabilités en fonction des zones géographiques du globe (zones extrêmement imprécises par définition) que la médecine états-unienne réinterprète en groupes ethno-raciaux. Beta-thalassémie est ainsi devenue aux États-Unis une maladie génétique dépistée principalement chez les Noirs car sa probabilité d'occurrence est forte en Afrique de l'Ouest. Or, tous les Noirs américains ne sont pas descendants de population d'Afrique de l'Ouest. De plus, cette maladie se retrouve aussi en Méditerranée et en Asie du Sudest. La médecine américaine opère ici un raccourci intellectuel entre zones géographiques, héritage génétique et races humaines qui renforce l'assise biologique de ces dernières. Pour une critique de l'usage racisant de la génétique cf. ZACK, Naomi, Philosophy of Science and Race, New York \& London, Routledge, 2002.

19. D'un point de vue épidémiologique ces groups englobent des populations extrêmement variées. L'identité ethno-raciale est perçue comme une donnée démographique et souvent comme le dénominateur le plus significatif. D'autres facteurs tels que l'activité sportive, les habitudes alimentaires, l'origine étrangère, la catégorie socioprofessionnelle, le niveau d'études, le revenu, l'absence ou le type d'assurance maladie, les données géographiques et les conditions de vie ne sont que rarement prises en compte, et donc très rarement recoupées avec l'identité ethno-raciale, et ce, même pour des études concernant des pathologies fortement influencées par les facteurs environnementaux. Pour plus de détails concernant les multiples facteurs masqués par l'utilisation des catégories raciales dans les études médicales cf. WILLIAMS, David R., LAVIZZO-MOUREY, Risa et WARREN, Rueben C., "The Concept of Race and Health Status in America ", Public Health Report, janvier-février 1994, vol. 109, 26-41.

20. SPICKARD, Paul R., "The Illogic of American Racial Categories ", in ROOT, Maria P., Racially Mixed People in America, Newbury Park, London, New Delhi, Sage Publications, 1992.

21. Tels que l'Anti-Defamation League et Asian Law Alliance.

22. Les organisations apportant leur soutien dans le guide officiel de l'électeur furent: l'Association des enseignants de Californie (California Teachers Association), la Fédération californienne des enseignants (California Federation of Teachers), la branche californienne de l'Association des parents d'élèves (California State PTA), l'Université de Californie (University of California ou UC) et Jack O'Conell le State Superintendent of Schools.

23. Le dernier exemple en date est la Proposition 11 censée, selon Arnold Schwarzenegger, régler le problème de gouvernance de l'État. Cet amendement à la constitution créa une commission indépendante constituée de citoyens ordinaires chargés de redécouper les circonscriptions électorales. Or, pour traiter un sujet aussi politique et aussi complexe en si peu de temps la commission a dû s'en remettre à des experts. Un tel sujet mériterait une réflexion de fond sur la crise de la représentation politique.

24. McGRIRR, op. cit., 2002.

25. Rappelons qu'après six longues semaines d'imbroglio juridique la Cour suprême avait ordonné l'arrêt du recompte de Floride, donnant ainsi la victoire à Georges W. Bush sur Al Gore, par 537 voix. La Cour avait estimé que les États n'étaient pas tenus de fournir des conditions uniformes en matière de système électoral. Cf. Bush v. Gore, 531 U.S. 98 (2000); Certains journalistes estiment, qu'en 2000, 40000 votes n'auraient pu être pris en considération, la grande majorité étant des bulletins de vote émanant de personnes afro-américaines ou hispaniques. Editorial, «The Recall Reargued », New York Times, 23 septembre 2003, A30.

26. Trois semaines avant l'échéance électorale, et alors que quelques 300000 personnes avaient déjà voté par procuration, la cour d'appel fédérale du $9^{\text {ème }}$ circuit suspendit l'élection jusqu'à nouvel ordre (proposant même de la repousser jusqu'en mars 2004) car la cour déclara, comme le soulignaient le Syndicat des libertés civiles (American Civil Liberties Union) et d'autres plaignants, que l'élection ne serait pas équitable de par la nature des bulletins de vote que $44 \%$ des 
Californiens devaient utiliser. MURPHY, Dean E., «California's Vote Delayed by Court over Punch Cards ", New York Times, 16 septembre 2003, A1 ; Les représentants de l'État de Californie ont euxmêmes reconnu que les anciennes machines à cartes perforées « engendrent plus d'erreurs que les nouveaux systèmes ".

27. MURPHY, Dean E., " Affirmative Action Foe's Latest Effort Complicates California Recall », New York Times, 3 août 2003, 1-13.

28. Même s'il est maintenant clair que les Hispaniques sont loin de représenter un bloc monolithique, en particulier sur les questions morales ou familiales pour lesquelles une partie de la communauté se positionne de façon traditionnelle et conservatrice. HENDRICKS, Tyche, "Latinos Activists gear up to register, get out vote ", San Francisco Chronicle, 13 août 2003, A16; Del OLMO, Franck, « Now Latinos Are in the Driver's Seat », Los Angeles Times, 27 juillet 2003, M5.

29. LeDUFF, Charlie, "Ethnic Issue in California Recall Play Out at Latino Parade ", New York Times, 8 septembre 2003, A19.

30. HADDOCK, Vicky, "Politics and Race", San Francisco Chronicle, 28 septembre 2003, p.D3; SCHRAG, Peter, "In California, White Men Are the Silent Plurality », New York Times, 14 septembre 2003, 3.

31. COILE, Zachary, "Question on NAACP boss' racism charge ", San Francisco Chronicle, 3 octobre 2003, A14.

32. SIMON, Mark, "Indian campaign donations in the spotlight », San Francisco Chronicle, 24 septembre 2003, A18.

33. TROUNSON, Rebecca, HELFAND, Duke \& BUSTILLO, Miguel, « Racial Data Measure May Be a Wild Card in Election", Los Angeles Times, 15 août 2003, A30; SCHRAG, Peter, "Race and gubernatorial recall: Can they be linked? ». Sacramento Bee, 30 juillet 2003, B7; SCHEVITZ, Tanya, « Recall's Strange Bedfellow », San Francisco Chronicle, 29 juillet 2003, A11.

34. Par exemple, la Proposition 54, qui fut présentée comme l'héritier de la Proposition 209, ne faisait même pas l'unanimité chez les auteurs de cette dernière. En effet, Glynn Custred (président du mouvement Americans Against Discrimination and Preference) et Thomas Wood (directeur de la très conservatrice California Association of Scholars) ne prirent pas du tout les mêmes positions vis-à-vis de la Proposition 54. Le premier y était favorable en tant que nouvelle pièce à l'édifice entamé par la Proposition 209; le second y était opposé justement parce que les statistiques ethno-raciales permettaient, selon lui, de s'assurer que la loi interdisant l'affirmative action ne soit pas détournée. Cf. NELSON, Jennifer, "A Reluctant No On 54: We Need The Boxes ", San Francisco Gate, 8 septembre 2003, Disponible à l'adresse: http://www.sfgate.com/cgi-bin/ article.cgi ?file =/gate/archive/2003/09/08/jnelson.DTL (page consultée le 23 avril 2009). (Notons au passage que Madame Nelson était l'attachée de presse de la campagne pour la Proposition 209 en 1996).

35. MORAIN, Dan, «Prop. 54 Sponsor Concedes Passage Is Now Unlikely », Los Angeles Times, 7 septembre 2003, A1.

36. Ward Connerly nous a confié dans un entretien qu'il recevait des coups de fils de représentants du Parti républicain lui expliquant qu'ils approuvaient sa démarche mais que pour des raisons électorales ils ne pouvaient l'appuyer publiquement. Entretien avec Ward CONNERLY, Directeur et fondateur du American Civil Rights Institute, le 31 août 2005 à Sacramento, CA.

37. En 2005, la Food And Drug Administration a autorisé la mise sur le marché du premier médicament pour traiter l'insuffisance cardiaque spécifiquement destiné aux «AfroAméricains ». RAHEMTULLA, Talsin \& BHOPA, Raj, « Pharmacogenetics and ethnically targeted therapies: New drug BiDil marks the return of biology to the debate aboutrace and ethnicity ", British Medical Journal, vol. 330, 7 mai 2005, 1036-37.

WADE, Nicholas, «Race Is Seen as Real Guide To Track Roots of Disease », New York Times, 30 juillet 2002, F1; « For Sale: A DNA Test To Measure Racial Mix », New York Times, 1er octobre 2002, 
F.4; «Scientists differ on genetics of race », International Herald Tribune, 28 octobre 2004, 6; « RaceBased Medicine Continued... ", New York Times, 14 novembre 2004, 4-12.

38. Pour plus de précisions sur ces questions cf. notamment MORNING, Ann, 2009, "Toward a Sociology of Racial Conceptualization for the 21st Century, Social Forces 87(3), 1167-92.

39. RAINE, George, «Bustamante airs first anti-Prop. 54 ad », San Francisco Chronicle, 18 septembre 2003, A12.

40. Une loi de 2000 qui fut adoptée suite à un référendum d'initiative populaire, Proposition 34, qui limitait les contributions aux candidats du vote en destitution à 21200 dollars par donneur.

41. Cette tactique allait s'avérer contre productive pour les tribus opérant les casinos. Les tribus $\mathrm{du}$ sud de la Californie étaient favorables à une participation active dans la campagne référendaire alors que les tribus du nord étaient plus réservées. Ces dernières avaient raison car les scandales du financement des campagnes ternirent leur image de "lobby exonéré d'impôts " et fournirent un argument de campagne de taille pour Schwarzenegger qui promit de réformer le statut des casinos amérindiens, notamment en les imposant fiscalement à la mesure de leurs bénéfices. WALTER, Dan, « Tribes lose high-stakes political gamble in recall election », Sacramento Bee, 20 octobre 2003, A3.

42. ROJAS, Aurelio, "Bustamante to shift disputed donations. The $\$ 3.8$ million move to fight Prop. 54 aims to quiet his critics ", Sacramento Bee, 7 septembre 2003, A1. La justice ordonna à Bustamante de ne pas utiliser cet argent même dans le cadre de la Proposition 54. Cf. BERTHELSEN, Christian, «Bustamante told not to use loophole », San Francisco Chronicle, 23 septembre 2003, A1. Ce dernier s'entêta pourtant et poursuivit sa campagne anti-Proposition 54 prétextant qu'il ne pouvait arrêter ses spots publicitaires sans rompre son contrat avec les chaînes de télévision, réduisant ainsi chaque jour les chances de la Proposition 54. Cf. MORAIN, Dan \& RUBIN, Joel, «Bustamante Ads Still on the Air ", Los Angeles Times, 25 septembre 2003, A26.

43. Les spots publicitaires sont incontournables lors des campagnes électorales dans un État aussi peuplé que la Californie. Dans des États plus petits les moyens plus traditionnels permettent de toucher une partie non négligeable de l'électorat.

44. COILE, Zachary, "Tribes add millions to McClintock, Bustamante», San Francisco Chronicle, 27 septembre 2003, A18.

45. Il reçut notamment des contributions significatives (1,7 millions de dollars) et secrètes de Rupert Murdoch, Joseph Coors ou encore Jerry Hume. Après que la Commission des pratiques politiques équitables (Fair Political Practice Commission), la même commission qui porta plainte contre Cruz Bustamante, ait porté plainte pour non divulgation des donations de campagne, ACRI dut payer 95000 dollars d'amende lors d'un règlement à l'amiable. BERTHELSEN, Christian, «Prop. 54's big-money backers revealed », San Francisco Chronicle, 19 mai 2005, B2.

46. WEBBER, Henry S., "The Failure of Health Care Reform: An Essay Review», The Social Service Review, vol. 69, n 2, juin 1995, 318.

47. Les minorités sont surreprésentées dans la population sans assurance maladie. U.S. CENSUS, "Income, Poverty, and Health Insurance Coverage in the United States: 2007», 2008, http:// www.census.gov/prod/2008pubs/p60-235.pdf (page consultée le 24 mai 2011).

48. Tous les opposants au vote de destitution ne manquèrent pas d'incorporer la Proposition 54 à leur combat contre les conservateurs, liant parfois directement le vote de destitution et la Proposition 54 à la lutte pour les droits civiques. SAMPLE, Herbert A., «Jesse Jackson fights recall and Prop. 54», Sacramento Bee, 17 septembre 2003, A3.

49. Pamphlet distribué par le ACLU de Californie du Sud, possession de l'auteur.

50. Editorial, "The San Francisco Chronicles Recommends/ PROP 54, Racial data: No ", San Francisco Chronicle, 5 octobre 2003, E8. Le Chronicle fut d'ailleurs précédé par le San Diego UnionTribune, qui est pourtant un journal beaucoup plus conservateur. Editorial, « No on Prop. 54; Poorly-crafted law is wrought with problems ", San Diego-Union Tribune, 29 septembre 2003, B6. 
51. Ce terme d'ignorance pour dénigrer l'absence de classification et de statistiques sera le leitmotiv de l'opposition à la Proposition 54. Cf. par exemple, BENNER, Laurence A., « Prop. 54 ; A dangerous veil of ignorance ", San Diego-Union Tribune, 1 octobre 2003, B7.

52. Cf. note 36 .

\section{RÉSUMÉS}

Aux États-Unis, les occasions de voir émerger un débat public sur la légitimité des classifications ethniques et/ou raciales de la population américaine sont extrêmement rares et ponctuelles. Toutefois, en 2003 , lors d'un référendum d'initiative populaire, les électeurs californiens ont pu se prononcer sur la question controversée de l'utilité d'une telle classification. Cette campagne électorale, à de nombreux égards atypique, nous éclaire tout de même sur les thèmes et enjeux du débat étatsunien concernant ces statistiques et les politiques publiques qui en dépendent. Mais surtout, elle illustre les difficultés à ouvrir un débat de fond sur la question de la taxonomie ethno-raciale. Cette question, d'un intérêt politique majeur, reste marquée par de profonds clivages partisans et, même lorsque l'occasion se présente, la société américaine s'avère peu disposée à se lancer dans une véritable réflexion sur la nature de la représentation identitaire et politique engendrée par les statistiques ethno-raciales.

In the U.S., public debates about the legitimacy of the classification of the American population along ethno-racial lines are quite rare. However, in 2003, voters in California had the opportunity to express their views on the necessity of this form of classification via referendum. This campaign was in many ways atypical but it still illustrates the complex and controversial nature of the debate about ethno-racial classification and the public policies relying on it. Moreover, it shows how difficult it is to launch a real political debate about ethno-racial taxonomy. This question is tangled in deep partisan divisions and, even when the opportunity presents itself, the American society seems unwilling to start any serious discussion about the very nature of the identities and the political representations created by ethno-racial statistics.

\section{AUTEUR}

\section{OLIVIER RICHOMME}

Université Lyon 2 